\title{
Oscillatory Blood Flow and Embolitic Plaque Effect Through a Microchannel with Metabolic Heat and Magnetic Field
}

\author{
K. W. Bunonyo and L. Ebiwareme
}

\begin{abstract}
An attempt was made to investigate an embolitic plaque effect on blood flow through a microchannel and the impact of the magnetic field, metabolic heat, and external heat source on improving blood flow. To address the aforementioned objectives, mathematical models were developed for blood flow and heat transfer with a source. The governing models were scaled using the dimensionless quantities, and the plaque area was derived from Dominguez [28], in which it was incorporated into the governing equations. The governing equations were further reduced to ordinary differential equations using the perturbation method, and the subsequent ordinary differential equations were solved using the method of undermined coefficients, and the constants obtained with the help of the matrix method using the boundary conditions. Furthermore, simulation was carried out to study the effect of the pertinent parameters using Wolfram Mathematica, a computational software. From the simulated results, it is seen that the entering parameters such as magnetic field parameter, the Reynolds number, Womersley number, oscillatory frequency parameter, and permeability parameter all affect the blood velocity and temperature profiles, showing significant impactful results that are useful to both mathematicians and clinicians.
\end{abstract}

Keywords - Embolic, Metabolism, Blood, Magnetic field, Plaque, Oscillatory.

\section{INTRODUCTION}

The embolic plaque buildup inside arteries raises the risk of developing cardiovascular disease such as heart attack, stroke, or cholesterol embolism. A cholesterol embolism, also called an atheroembolism or cholesterol embolization syndrome, is when a crystal of cholesterol breaks off a plaque deposit inside one of the arteries. This cholesterol crystal can then travel through your bloodstream and block blood flow in one of the smaller blood vessels or microchannels, causing inflammation. The blockage and inflammation can damage organs or tissues that are supplied by blood vessels. The most commonly affected organs are the kidneys, skin, gastrointestinal system, and the brain, Ghanem et al. [1].

The cardiovascular system is made up of blood cells, blood vessels, and the heart. The main function of the heart is to pump blood into circulation, to the tissues and organs of the human body through the blood vessels. According to Bunonyo and Amos [2], blood is an essential ingredient of the vitality of the body system, and its major constituents are the red blood cells (erythrocytes), white blood cells (leukocytes), platelets, and plasma fluid. Blood contains hemoglobin, which has magnetic properties that are different depending on the oxidation state of hemoglobin. The body contains proteins, such as LDL cholesterol, which when in increasing quantity in the cardiovascular system can build up in various arteries, clogging and reducing their flexibility. Atherosclerosis results in restricted normal blood flow because of the loss of vessel flexibility, so the heart works harder to push blood through to the downstream, Okpeta and Bunonyo [3].

Bio-magnetic fluid dynamics has many major applications, such as magnetic drug targeting, adjusting blood flow during surgery, transporting complex bio-waste fluids, cancer tumor treatment, etc. Extensive research has been undertaken on the fluid dynamics of bio-magnetic fluids under the presence of an external magnetic field. The application of magneto-hydrodynamics in physiological flow is of growing interest as many researchers have reported that blood is an electrically conducting fluid, Singh and Rathee [4]. Over several decades, there have been so many researchers that have worked on blood flow problems; a mathematical modeling of tumor growth in mice following low-level direct electric current was proposed by Cabrales et al. [5].

Submitted on December 28, 2021

Published on January 19, 2022.

K. W. Bunonyo, Department of Mathematics and Statistics, Federal University Otuoke, Ogbia, Nigeria.

(corresponding e-mail: wilcoxbk@fuotuoke.edu.ng)

L. Ebiwareme, Department of Mathematics, Rivers State University, Port Harcourt, Nigeria 
A study was carried out on a nonlinear fractional mathematical model of tumor cells in the presence of chemotherapeutic treatment by Kumar and Atangana [6].

An inverse problem formulation for parameter estimation of a reaction diffusion model of low grade gliomas was carried out by Gholami et al. [7]. They presented a numerical scheme for solving a parameter estimation problem for a model of low-grade glioma growth. They estimated the spatial distribution of tumor concentration as well as the magnitude of anisotropic tumor diffusion.

Molloy and Markus [8] studied whether asymptomatic embolization predicts stroke and TIA risk in patients with carotid artery stenosis. The method is an improved method of identifying patients at high risk of thromboembolism, which allows for an improved target of therapy.

Bru and Herrero [9] studied the physical laws of tumor growth and modeled cancer processes. Their model describes the growth of solid tumors, which provides a procedure to extract information about the growth dynamics from the analysis of the geometrical properties of the interface tumor-host tissue.

Dai and Liu [10] proposed optimal control and pattern formation for a haptotaxis model of solid tumor invasion. The results of their work improved and partially extended the previous results defined in their work.

Duchting and Vogelsaenger [11] proposed aspects of modeling and simulating tumor growth and treatment. The model is based on the hypothesis that the proliferation of malignant cells may be simulated by an unstable closed-loop control circuit. Duchting and Vogelsaenger [12] and [13] proposed progress in modeling and simulation of three-dimensional tumor growth and treatment. Their work showed how tumor treatment may be optimized in the long run by using computer simulation experiments as a powerful new tool.

Hawkins-Daarud et al. [14] modeled tumor associated edema in gliomas during angiogenic therapy and its impact on manageable tumors. Gliomas are the most aggressive form of primary brain tumor. They looked at virtual tumors with different growth kinetics and discovered that tumors with lower proliferation rates had the greatest reduction in swelling from the treatments they used.

Makinde et al. [15] studied the blood vessels formed in asthmatic airways that are involved in inflammatory and airway remodeling processes in chronic asthma. Vascular endothelial cell growth factor (VEGF) and angiopoietin-1 (Ang-1) are primary angiogenic growth factors, involved in the formation of such blood vessels. VEGF has been reported to contribute to non-specific airway hyper-responsiveness, have chemotactic effects on eosinophils, and enhance airway smooth muscle cell proliferation. Makinde and Osalusi [16] researched the hydromagnetic steady flow of a viscous conducting fluid in a channel with slip at the permeable boundaries. An analytical solution was obtained for the governing nonlinear boundary values problem using the perturbation method together with a pade approximation technique based on computer extended series solution and their results.

Mandel [17] investigated an unsteady analysis of non-Newtonian blood through the tapered arteries with stenosis. The non-Newtonian blood flow problem was solved numerically with the finite difference scheme used to solve the unsteady nonlinear Navier-Stokes equation in the cylindrical coordinate system governing flow with a verifiable assumption to reduce the problem to two-dimensional flow. An iterative method has been shown to solve the equation numerically, and it is known that the vascular wall deformability and non-Newtonian characteristics of the following blood affect the axial velocity profile.

Mathematical modeling, analysis, and simulation of tumor dynamics with drug interventions were carried out by Unni and Seshaiyer [18]. Their results suggest that the model employed is a robust candidate for studying the dynamics of tumor cells and helps to provide the dynamic interactions between the tumor cells, immune system, and drug-response systems.

Miklavcic et al. [19] carried out mathematical modeling of tumor growth in mice following electrotherapy and bleomycin treatment. The effect of bleomycin on tumor growth was obtained by introducing the influential parameter which transferred the bleomycin concentration in tumor tissue obtained from the pharmacokinetic model to the effect on tumor growth.

The modelling of tumor cell regression in response to chemotherapeutic treatment was carried out by Ansarizadeh et al. [20]. They observed that the response of three different levels of immune system strength to the pulsed chemotherapy was better if a chemotherapeutic drug was injected near the invasive fronts of the tumor. Morales-Delgado et al. [21] proposed an application of the Caputo-Fabrizio and Atangana-Baleanu fractional derivatives to a mathematical model of the chemotherapy effect. They obtained approximate analytical solutions of a cancer chemotherapy effect model involving fractional derivatives with an exponential kernel and with the general Mittag-Leffler function.

Stochastic modeling of tumor growth within an organ during chemotherapy using bivariate birth, death, and migration processes was carried out by Padi et al. [22]. Their models are derived from stochastic differential equations, and model behavior was analyzed with numerical data.

The development of a hybrid cellular automaton model to mimic the growth of avascular tumors, including the infusion of a bioreductive drug to study the effects of protein binding on drug transportation, was reported by Kazmi et al. [23]. They conducted experiments with multicellular tumor 
spheroids and the results showed good agreement with their predicted growth dynamics. Zhu et al. [24] investigated tumor growth under hyperthermia conditions and came to a conclusion with both the tumor growth rate curve and corresponding average glucose concentration, obtaining numerical results illustrating the controlling effect on tumor growth under hyperthermia conditions in the early stage.

Our objective in the present research is to investigate whether metabolic heat, magnetic field intensity, and heat from a source contribute to an oscillatory blood flow with an embolitic plaque through a microchannel. The challenge in mathematical modeling lies in the description of the biophysical phenomenon accurately. This present paper is an extension of the previous work by Bunonyo and Amadi [25].

\section{MATHEMATICAL FORMULATION}

This study focused on investigating oscillatory blood flow with embolitic plaque through a microchannel with metabolic heat and a magnetic field. The study entails mathematical formulation of the biophysical problem by extending the Navier-Stoke equation to represent the blood flow and formulating an energy equation by modifying the bio-heat equations with sources such as metabolic and other source terms. Blood is considered an incompressible, viscous, and electrically conducting fluid. The channel is assumed to be porous and permeable, and a magnetic field is applied externally and vertically to it while it is moving in a horizontal direction. The channel is assumed to be Cartesian in two dimensions.

\section{GOVERNING EQUATION}

Following Misra and Adhikary [26], Eldesoky [27], Bunonyo and Amos [2], and Bunonyo and Amadi [25], we present the blood momentum equation and the heat equation with source terms governing the flow as follows:

$$
\begin{aligned}
& \frac{\partial w^{*}}{\partial t^{*}}+V_{0}\left(1+\varepsilon e^{i \omega t}\right) \frac{\partial w^{*}}{\partial y^{*}}=\frac{1}{\rho_{b}} \frac{\partial P^{*}}{\partial x^{*}}+v \frac{\partial^{2} w^{*}}{\partial y^{* 2}}-\frac{v w^{*}}{k^{*}}-\sigma \frac{B_{0}^{2} w^{*}}{\rho_{b}}+g \beta_{T b}\left(T-T_{a}\right) \\
& \rho_{b} c_{b} \frac{\partial T^{*}}{\partial t^{*}}=k_{b} \frac{\partial^{2} T^{*}}{\partial y^{* 2}}-\rho_{a} \rho_{b} w_{b}\left(T-T_{a}\right)+S
\end{aligned}
$$

Following Bunonyo and Amos [2], we consider the region of the tumor growth is formulated as follows:

$$
y^{*}= \begin{cases}R_{0}-\delta^{*} \cos \left(2 \pi \frac{x^{*}}{\lambda^{*}}\right) & \text { at } 0 \leq x^{*} \leq d_{0} \\ R_{0} & \text { at } d_{0} \leq x^{*} \leq \lambda^{*}\end{cases}
$$

where

$$
\delta=\delta_{0} e^{\left(\frac{\lambda_{1}\left(1-e^{\left(-\alpha_{2} t\right.}\right)}{\alpha_{2}}\right)},
$$

according to Dominguez [28]

The corresponding boundary conditions are:

$$
\left.\begin{array}{llrl}
\frac{\partial w^{*}}{\partial y^{*}}=0, \frac{\partial T^{*}}{\partial y^{*}}=0 & \text { at } & y^{*}=0 \\
w^{*}=0, T^{*}=0 & \text { at } & y^{*}=R
\end{array}\right\}
$$




\section{DIMENSIONLESS PARAMETERS}

We introduce the following dimensionless parameters:

$$
\begin{aligned}
& \left.y=\frac{y^{*}}{R_{0}}, x=\frac{x^{*}}{\lambda}, \lambda=\frac{\lambda^{*}}{R_{0}}, \delta=\frac{\delta^{*}}{R_{0}}, w=\frac{w^{*}}{v w_{0}}, P=\frac{P^{*} R_{0}^{2}}{\mu \lambda w_{0}}, \theta=\frac{T-T_{a}}{T_{w}-T_{a}}, R d_{1}=\frac{R_{0}^{2} \rho_{a} \rho_{b} F}{\mu_{b} c_{b}}, G r=\frac{g \beta_{T b}\left(T_{w}-T_{a}\right) R_{0}^{2}}{v^{2} w_{0}}\right) \\
& \frac{R_{0}^{2}}{k^{*}}=\frac{1}{k}, \operatorname{Re}=\frac{V_{0} R_{0}}{v}, \alpha^{2}=\frac{\rho_{b} R_{0}^{2} \omega}{\mu}, M^{2}=\frac{\sigma B_{0}^{2} R_{0}^{2}}{\mu}, t=\omega t^{*}, \operatorname{Pr}=\frac{\mu_{b} c_{b}}{k_{b}}, S_{1}=\frac{R_{0}^{2} S(t)}{k_{b}\left(T_{w}-T_{a}\right)}
\end{aligned}
$$

Using the dimensionless variables in equation (5), the momentum equation governing the flow is reduced to:

$$
\begin{aligned}
& \alpha^{2} \frac{\partial w}{\partial t}+\operatorname{Re}\left(1+\varepsilon e^{i \omega t}\right) \frac{\partial w}{\partial y}=\frac{\partial P}{\partial x}+\frac{\partial^{2} w}{\partial y^{2}}-\frac{w}{k}-M^{2} w+G r \theta \\
& \alpha^{2} \operatorname{Pr} \frac{\partial \theta}{\partial t}=\frac{\partial^{2} \theta}{\partial y^{2}}-\operatorname{Rd}_{1} \operatorname{Pr} \theta+S_{1}
\end{aligned}
$$

The region of tumor region is reduced to:

$$
\begin{aligned}
& y= \begin{cases}1-\delta \cos (2 \pi x) & \text { at } 0 \leq x \leq \frac{d_{0}}{\lambda} \\
1 & \text { at } \frac{d_{0}}{\lambda} \leq x \leq R_{0}\end{cases} \\
& \delta=\delta_{0} e^{\left(\frac{\lambda_{1}\left(1-e^{\left(-\alpha_{2} t\right.}\right)}{\alpha_{2}}\right)}
\end{aligned}
$$

The corresponding boundary conditions are:

$$
\left.\begin{array}{lll}
\frac{\partial w}{\partial y}=0, \frac{\partial \theta}{\partial y}=0 & \text { at } & y=0 \\
w=0, \theta=0 & \text { at } & y=1-\delta \cos (2 \pi x)
\end{array}\right\}
$$

\section{Method of SOlution}

Since the flow by the pumping action of the ventricles, we assume the solution to be in the following form:

$$
\begin{aligned}
& w(y, t)=w_{0}(y)+w_{1}(y) e^{i \omega t} \\
& \theta(y, t)=\theta_{0}(y)+\theta_{1}(y) e^{i \omega t} \\
& S_{1}=S_{2} e^{i \omega t} \\
& -\frac{\partial P}{\partial x}=P_{0}+P_{1} e^{i \omega t}
\end{aligned}
$$

Using equation (10) to simplify equation (6) - (9), we have:

Steady State for which $\varepsilon=0$, the dimensionless momentum equation is:

$$
\begin{aligned}
& \frac{\partial^{2} w_{0}}{\partial y^{2}}-\operatorname{Re}\left(1+\varepsilon e^{i \omega t}\right) \frac{\partial w_{0}}{\partial y}-\beta_{0}^{2} w_{0}=-P_{0}-G r \theta_{0} \\
& \frac{\partial^{2} \theta_{0}}{\partial y^{2}}-R_{1} \operatorname{Pr} \theta_{0}=0
\end{aligned}
$$


The oscillatory state which $\varepsilon \neq 0$, dimensionless momentum equation is:

$$
\begin{aligned}
& \frac{\partial^{2} w_{1}}{\partial y^{2}}-\operatorname{Re}\left(1+\varepsilon e^{i \omega t}\right) \frac{\partial w_{1}}{\partial y}-\beta_{1}^{2} w_{1}=-P_{1}-G r \theta_{1} \\
& \frac{\partial^{2} \theta_{1}}{\partial y^{2}}-\left(R d_{1}+\alpha^{2} i \omega\right) \operatorname{Pr} \theta_{1}=-S_{2}
\end{aligned}
$$

The region of atherosclerosis is reduced to:

$$
\begin{aligned}
& y= \begin{cases}1-\delta \cos (2 \pi x) & \text { at } 0 \leq x \leq \frac{d_{0}}{\lambda} \\
1 & \text { at } \frac{d_{0}}{\lambda} \leq x \leq R_{0}\end{cases} \\
& \delta=\delta_{0} e^{\left(\frac{\lambda_{1}\left(1-e^{(-\alpha t)}\right)}{\alpha_{2}}\right)} \\
& \lambda_{1}=-0.03, \alpha_{2}=0.3, t=5, \delta_{0}=2, x=0.13
\end{aligned}
$$

The corresponding boundary conditions are:

$$
\left.\begin{array}{lll}
\frac{\partial w_{0}}{\partial y}=0, \frac{\partial w_{1}}{\partial y}=0 \& \frac{\partial \theta_{0}}{\partial y}=0, \frac{\partial \theta_{1}}{\partial y}=0 & \text { at } & y=0 \\
w_{0}=0, w_{1}=0 \& \theta_{0}=0, \theta_{1}=0 & \text { at } & y=1-\delta \cos (2 \pi x)+R_{T}
\end{array}\right\}
$$

where $\beta_{1}^{2}=\left(\frac{1}{k}+M^{2}+\alpha^{2} i \omega\right), \beta_{0}^{2}=\left(\frac{1}{k}+M^{2}\right)$.

Solving equation (3.14), we let $\theta_{0}=e^{m_{0} y}$, we obtain the homogenous solution as follows:

$$
\theta_{0}(y)=0
$$

Substitute equation (20) into equation (13), we have the characteristic equation as:

$$
p_{0}\left(m_{1}\right)=m_{1}^{2}-\operatorname{Rem}_{1}-\beta_{0}^{2}
$$

The roots of the equation (21) are:

$$
m_{1}=\frac{R e+\sqrt{R e^{2}+4 \beta_{0}^{2}}}{2} \text { and } m_{2}=\frac{R e-\sqrt{R e^{2}+4 \beta_{0}^{2}}}{2}
$$

Then the general solution of equation (13) is:

$$
w_{0}(y)=A_{1} e^{m_{1} y}+B_{1} e^{m_{2} y}+\frac{P_{0}}{\beta_{0}^{2}},
$$

Using the boundary conditions in equation (19) to resolve the coefficients in equation (22), we have:

$$
\begin{aligned}
& A_{1}\left(\frac{R e+\sqrt{R e^{2}+4 \beta_{0}^{2}}}{2}\right)+B_{1}\left(\frac{R e-\sqrt{R e^{2}+4 \beta_{0}^{2}}}{2}\right)=0, \\
& A_{1} e^{\left(\frac{R e+\sqrt{R e^{2}+4 \beta_{0}^{2}}}{2}\right) h}+B_{1} e^{\left(\frac{R e-\sqrt{R e^{2}+4 \beta_{0}^{2}}}{2}\right) h}=-\frac{P_{0}}{\beta_{0}^{2}},
\end{aligned}
$$

We can transform equation (23) and (24) into a matrix as follows: 


$$
\left(\begin{array}{cc}
\left(\frac{R e+\sqrt{R e^{2}+4 \beta_{0}^{2}}}{2}\right) & \left(\frac{R e-\sqrt{R e^{2}+4 \beta_{0}^{2}}}{2}\right) \\
e^{\left(\frac{R e+\sqrt{R e^{2}+4 \beta_{0}^{2}}}{2}\right) h} & e^{\left(\frac{R e-\sqrt{R e^{2}+4 \beta_{0}^{2}}}{2}\right) h}
\end{array}\right)=\left(\begin{array}{c}
A_{1} \\
B_{1}
\end{array}\right)=\left(\begin{array}{c}
0 \\
-\frac{P_{0}}{\beta_{0}^{2}}
\end{array}\right),
$$

Solving the matrix in equation (25), we have the following constant coefficients as follows:

$$
\begin{aligned}
& A_{1}=\frac{m_{2} P_{0}}{\beta_{0}^{2}}\left(\frac{1}{m_{1} e^{m_{2} h}-e^{m_{1} h} m_{2}}\right) \\
& B_{1}=\frac{m_{1} P_{0}}{m_{2} \beta_{0}^{2}}\left(\frac{1}{e^{m^{m} h}-\frac{m_{1}}{m_{2}} e^{m_{2} h}}\right)
\end{aligned}
$$

So that equation (22) becomes:

$$
w_{0}(y)=\frac{m_{2} P_{0}}{\beta_{0}^{2}}\left(\frac{1}{m_{1} e^{m_{2} h}-e^{m_{1} h} m_{2}}\right) e^{m_{1} y}+\frac{m_{1} P_{0}}{m_{2} \beta_{0}^{2}}\left(\frac{1}{e^{m_{1} l_{1}}-\frac{m_{1}}{m_{2}}}\right) e^{m_{2} y}+\frac{P_{0}}{\beta_{0}^{2}},
$$

Recalling equation (16), we have:

$\frac{\partial^{2} \theta_{1}}{\partial y^{2}}-\beta_{3}^{2} \theta_{1}=-S_{2}$

where $\beta_{3}^{2}=\left(R d_{1}+\alpha^{2} i \omega\right) \operatorname{Pr}$.

Solving equation (29), we have the following:

$\theta_{1}(y)=A_{2} e^{\beta_{3} y}+B_{2} e^{-\beta_{3} y}+\frac{S_{2}}{\beta_{3}^{2}}$,

where, $m_{3}=\beta_{3}$ and $m_{4}=-\beta_{3}$.

Using the boundary conditions in equation (19) to resolve the coefficients in equation (30), we have:

$$
\begin{aligned}
& A_{2} \beta_{3}-B_{2} \beta_{3}=0 \\
& A_{2} e^{\beta_{3} h}+B_{2} e^{-\beta_{3} h}=-\frac{S_{2}}{\beta_{3}^{2}},
\end{aligned}
$$

We can transform equation (31) into a matrix as follows:

$$
\begin{aligned}
& \left(\begin{array}{cc}
\beta_{3} & -\beta_{3} \\
e^{\beta_{3} h} & e^{-\beta_{3} h}
\end{array}\right)\left(\begin{array}{l}
A_{2} \\
B_{2}
\end{array}\right)=\left(\begin{array}{c}
0 \\
-\frac{S_{2}}{\beta_{3}^{2}}
\end{array}\right), \\
& \left.\left.\Delta_{0}=\left|\begin{array}{cc}
\beta_{3} & -\beta_{3} \\
e^{\beta_{3} h} & e^{-\beta_{3} h}
\end{array}\right|, \Delta_{1}=\mid \begin{array}{cc}
0 & -\beta_{3} \\
-\frac{S_{2}}{\beta_{3}^{2}} & e^{-\beta_{3} h}
\end{array}\right), \Delta_{2}=\mid \begin{array}{cc}
\beta_{3} & 0 \\
e^{\beta_{3} h} & -\frac{S_{2}}{\beta_{3}^{2}}
\end{array}\right),
\end{aligned}
$$

Solving equation (32), we have the following constant coefficients as follows: 


$$
\begin{aligned}
& A_{2}=-\frac{S_{2}}{\beta_{3}^{2}\left(e^{-\beta_{3} h}+e^{\beta_{3} h}\right)} \\
& B_{2}=-\frac{S_{2}}{\beta_{3}^{2}\left(e^{-\beta_{3} h}+e^{\beta_{3} h}\right)}
\end{aligned}
$$

If can substitute equation (34) and (35) into equation (30), we have:

$$
\theta_{1}(y)=\left(-\frac{S_{2}}{\beta_{3}^{2}\left(e^{-\beta_{3} h}+e^{\beta_{3} h}\right)}\right) e^{\beta_{3} y}-\left(\frac{S_{2}}{\beta_{3}^{2}\left(e^{-\beta_{3} h}+e^{\beta_{3} h}\right)}\right) e^{-\beta_{3} y}+\frac{S_{2}}{\beta_{3}^{2}},
$$

We can recall equation (15) as follows:

$$
\frac{\partial^{2} w_{1}}{\partial y^{2}}-\operatorname{Re}\left(1+\varepsilon e^{i \omega t}\right) \frac{\partial w_{1}}{\partial y}-\beta_{1}^{2} w_{1}=-P_{1}-G r \theta_{1}
$$

Substitute equation (36) into equation (37), which is:

$$
\frac{\partial^{2} w_{1}}{\partial y^{2}}-R e\left(1+\varepsilon e^{i \omega t}\right) \frac{\partial w_{1}}{\partial y}-\beta_{1}^{2} w_{1}=-\left(P_{1}+\frac{S_{2}}{\beta_{3}^{2}}\right)+\left(\frac{S_{2} G r}{\beta_{3}^{2}\left(e^{-\beta_{3} h}+e^{\beta_{3} h}\right)}\right) e^{\beta_{3} y}+\left(\frac{S_{2} G r}{\beta_{3}^{2}\left(e^{-\beta_{3} h}+e^{\beta_{3} h}\right)}\right) e^{-\beta_{3} y}
$$

Solving equation (38), we let $w_{1}=e^{m_{11} y}$, so that we have the characteristics equation as:

$$
p_{01}\left(m_{11}\right)=m_{11}^{2}-\alpha_{1} m_{11}-\beta_{1}^{2}
$$

where $\alpha_{1}=\operatorname{Re}\left(1+\varepsilon e^{i \omega t}\right)$.

The roots of the equation (39) are:

$$
m_{11}=\frac{\alpha_{1}+\sqrt{\alpha_{1}^{2}+4 \beta_{1}^{2}}}{2} \text { and } m_{21}=\frac{\alpha_{1}-\sqrt{\alpha_{1}^{2}+4 \beta_{1}^{2}}}{2}
$$

Thence, the complimentary solution of equation (38) is:

$$
w_{c}(y)=A_{4} e^{m_{11} y}+B_{4} e^{m_{21} y},
$$

The particular solution of equation (38) is:

$$
w_{p}=\frac{P_{2}}{\beta_{1}^{2}}+\left(\frac{P_{3}}{\beta_{3}^{2}-\alpha_{1} \beta_{3}-\beta_{1}^{2}}\right) e^{\beta_{3} y}+\left(\frac{P_{3}}{\beta_{3}^{2}-\alpha_{1} \beta_{3}-\beta_{1}^{2}}\right) e^{-\beta_{3} y}
$$

So, the general solution to equation (38) is:

$$
\begin{aligned}
& w_{1}(y)=A_{4} e^{m_{11} y}+B_{4} e^{m_{21} y}+\frac{P_{2}}{\beta_{1}^{2}}+\left(\frac{P_{3}}{\beta_{3}^{2}-\alpha_{1} \beta_{3}-\beta_{1}^{2}}\right) e^{\beta_{3} y}+\left(\frac{P_{3}}{\beta_{3}^{2}-\alpha_{1} \beta_{3}-\beta_{1}^{2}}\right) e^{-\beta_{3} y}, \\
& \text { where } A_{0}=\frac{P_{2}}{\beta_{1}^{2}}, A=\left(\frac{P_{3}}{\left(\beta_{3}^{2}-\alpha_{1} \beta_{3}-\beta_{1}^{2}\right)}\right), B=\frac{P_{3}}{\left(\beta_{3}^{2}-\alpha_{1} \beta_{3}-\beta_{1}^{2}\right)} \\
& \alpha_{1}=\operatorname{Re}\left(1+\varepsilon e^{i \omega t}\right), P_{2}=\left(P_{1}+\frac{S_{2}}{\beta_{3}^{2}}\right), P_{3}=\left(\frac{S_{2} G r}{\beta_{3}^{2}\left(e^{-\beta_{3} h}+e^{\beta_{3} h}\right)}\right)
\end{aligned}
$$

Solving equation (42) using the boundary condition in equation (19), we have: 


$$
\begin{aligned}
& A_{4} m_{11}+B_{4} m_{21}=0 \\
& A_{4} e^{m_{11} h}+B_{4} e^{m_{21} h}=\left(\frac{P_{3}}{\beta_{3}^{2}+\alpha_{1} \beta_{3}-\beta_{1}^{2}}\right) e^{\beta_{3} h}+\left(\frac{P_{3}}{\beta_{3}^{2}+\alpha_{1} \beta_{3}+\beta_{1}^{2}}\right) e^{-\beta_{3} h}-\frac{P_{2}}{\beta_{1}^{2}}
\end{aligned}
$$

Equation (43) can also be written as follows:

$$
\begin{aligned}
& A_{4}\left(\frac{\alpha_{1}+\sqrt{\alpha_{1}^{2}+4 \beta_{1}^{2}}}{2}\right)+B_{4}\left(\frac{\alpha_{1}-\sqrt{\alpha_{1}^{2}+4 \beta_{1}^{2}}}{2}\right)=0, \\
& A_{4} e^{\left(\frac{\alpha_{1}+\sqrt{\alpha_{1}^{2}+4 \beta_{1}^{2}}}{2}\right) h}+B_{4} e^{\left(\frac{\alpha_{1}-\sqrt{\alpha_{1}^{2}+4 \beta_{1}^{2}}}{2}\right) h}=\left(\frac{P_{3}}{\beta_{3}^{2}+\alpha_{1} \beta_{3}-\beta_{1}^{2}}\right) e^{\beta_{3} h}+\left(\frac{P_{3}}{\beta_{3}^{2}+\alpha_{1} \beta_{3}+\beta_{1}^{2}}\right) e^{-\beta_{3} h}-\frac{P_{2}}{\beta_{1}^{2}},
\end{aligned}
$$

Equations (43), (44) and (45) can be transformed into a matrix as follows:

$$
\begin{aligned}
& \left(\begin{array}{cc}
m_{11} & m_{21} \\
e^{m_{11} h} & e^{m_{21} h}
\end{array}\right)\left(\begin{array}{l}
A_{4} \\
B_{4}
\end{array}\right)=\left(\begin{array}{c}
0 \\
P_{4}
\end{array}\right) \\
& \left(\begin{array}{cc}
\left(\frac{\alpha_{1}+\sqrt{\alpha_{1}^{2}+4 \beta_{1}^{2}}}{2}\right) & \left.\left(\frac{\alpha_{1}-\sqrt{\alpha_{1}^{2}+4 \beta_{1}^{2}}}{2}\right)\right)\left(\begin{array}{c}
A_{4} \\
e^{\left(\frac{\left.\alpha_{1}+\sqrt{\alpha_{1}^{2}+4 \beta_{1}^{2}}\right)}{2}\right)}
\end{array} e^{\left(\frac{\alpha_{1}-\sqrt{\alpha_{1}^{2}+4 \beta_{1}^{2}}}{2}\right) h}\right.
\end{array}\right)=\left(\begin{array}{c}
0 \\
B_{4}
\end{array}\right),
\end{aligned}
$$

Solving for the constants in equation (45), we have the following:

$$
A_{4}=\frac{m_{21} P_{4}}{m_{21} e^{m_{11} h}-m_{11} e^{m_{21} h}}, B_{4}=\frac{m_{11} P_{4}}{m_{11} e^{m_{21} h}-m_{21} e^{m_{11} h}}
$$

The blood velocity profile is with the effect of heat and metabolic heat is obtained after we substituted equation (42) and (28) into equation (12), we have:

$$
w(y, t)=\left(\begin{array}{l}
\frac{m_{2} P_{0}}{\beta_{0}^{2}}\left(\frac{1}{m_{1} e^{m_{2} h}-e^{m_{1} h} m_{2}}\right) e^{m_{1} y}+\frac{m_{1} P_{0}}{m_{2} \beta_{0}^{2}}\left(\frac{1}{e^{m_{1} h}-\frac{m_{1}}{m_{2}} e^{m_{2} h}}\right) e^{m_{2} y}+\frac{P_{0}}{\beta_{0}^{2}}+A_{4} e^{m_{11} y} \\
+B_{4} e^{m_{21} y}+\frac{P_{2}}{\beta_{1}^{2}}+\left(\frac{P_{3}}{\beta_{3}^{2}-\alpha_{1} \beta_{3}-\beta_{1}^{2}}\right) e^{\beta_{3} y}+\left(\frac{P_{3}}{\beta_{3}^{2}-\alpha_{1} \beta_{3}-\beta_{1}^{2}}\right) e^{-\beta_{3} y}
\end{array}\right) e^{i \omega t},
$$

The temperature profile is obtained after we have substituted equation (36) and equation (20) into equation (12), we have:

$$
\begin{aligned}
& \theta(y, t)=\left(\left(-\frac{S_{2}}{\beta_{3}^{2}\left(e^{-\beta_{3} h}+e^{\beta_{3} h}\right)}\right) e^{\beta_{3} y}-\left(\frac{S_{2}}{\beta_{3}^{2}\left(e^{-\beta_{3} h}+e^{\beta_{3} h}\right)}\right) e^{-\beta_{3} y}+\frac{S_{2}}{\beta_{3}^{2}}\right) e^{i \omega t}, \\
& \text { where } A_{0}=\frac{P_{2}}{\beta_{1}^{2}}, A=\left(\frac{P_{3}}{\left(\beta_{3}^{2}-\alpha_{1} \beta_{3}-\beta_{1}^{2}\right)}\right), B=\frac{P_{3}}{\left(\beta_{3}^{2}-\alpha_{1} \beta_{3}-\beta_{1}^{2}\right)}, A_{4}=\frac{m_{21} P_{4}}{m_{21} e^{m_{11} h}-m_{11} e^{m_{21} h}}, \\
& B_{4}=\frac{m_{11} P_{4}}{m_{11} e^{m_{21} h}-m_{21} e^{m_{11} h}}, \alpha_{1}=\operatorname{Re}\left(1+\varepsilon e^{i \omega t}\right), P_{2}=\left(P_{1}+\frac{S_{2}}{\beta_{3}^{2}}\right), P_{3}=\left(\frac{S_{2} G r}{\beta_{3}^{2}\left(e^{-\beta_{3} h}+e^{\beta_{3} h}\right)}\right),
\end{aligned}
$$




$$
\begin{aligned}
& P_{4}=\left(\frac{P_{3}}{\beta_{3}^{2}+\alpha_{1} \beta_{3}-\beta_{1}^{2}}\right) e^{\beta_{3} h}+\left(\frac{P_{3}}{\beta_{3}^{2}+\alpha_{1} \beta_{3}+\beta_{1}^{2}}\right) e^{-\beta_{3} h}-\frac{P_{2}}{\beta_{1}^{2}}, \beta_{3}^{2}=\left(R d_{1}+\alpha^{2} i \omega\right) P r \\
& m_{11}=\frac{\alpha_{1}+\sqrt{\alpha_{1}^{2}+4 \beta_{1}^{2}}}{2} \text { and } m_{21}=\frac{\alpha_{1}-\sqrt{\alpha_{1}^{2}+4 \beta_{1}^{2}}}{2}, \beta_{1}^{2}=\left(\frac{1}{k}+M^{2}+\alpha^{2} i \omega\right), \beta_{0}^{2}=\left(\frac{1}{k}+M^{2}\right) \\
& m_{1}=\frac{R e+\sqrt{R e^{2}+4 \beta_{0}^{2}}}{2} \text { and } m_{2}=\frac{R e-\sqrt{\operatorname{Re}^{2}+4 \beta_{0}^{2}}}{2}
\end{aligned}
$$

\section{RESULTS AND DISCUSSION}

We coded equations (48) and (49) using Wolfram Mathematica software and simulated them by varying the pertinent parameters to study the effect of the parameters on the blood velocity profile and temperature profile. The parameter values are obtained from Bunonyo and Amos [2], Misra and Adhikary [26], Eldesoky [27], and Bunonyo and Amadi [25]. The simulated results are labeled Fig. 1 to Fig. 14, with the varying parameters on the legend indicating the entering effect on the flow profiles

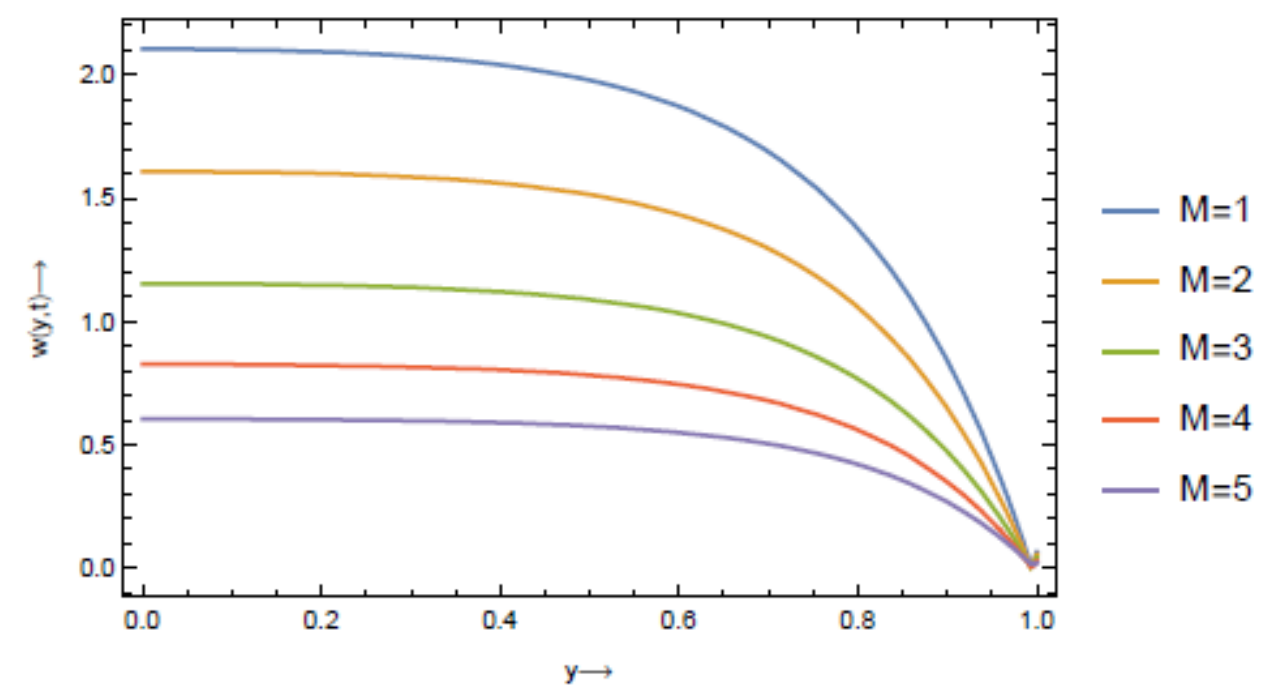

Fig. 1. Effect of Magnetic field on Velocity Profile $w(y, t)$, with $k=0.05, \alpha=0.2, \omega=3, \varepsilon=0.02, \operatorname{Pr}=21, R d_{1}=0.4, S_{2}=5, t=5 R e=0.5, R_{T}=0.5, G r=15$

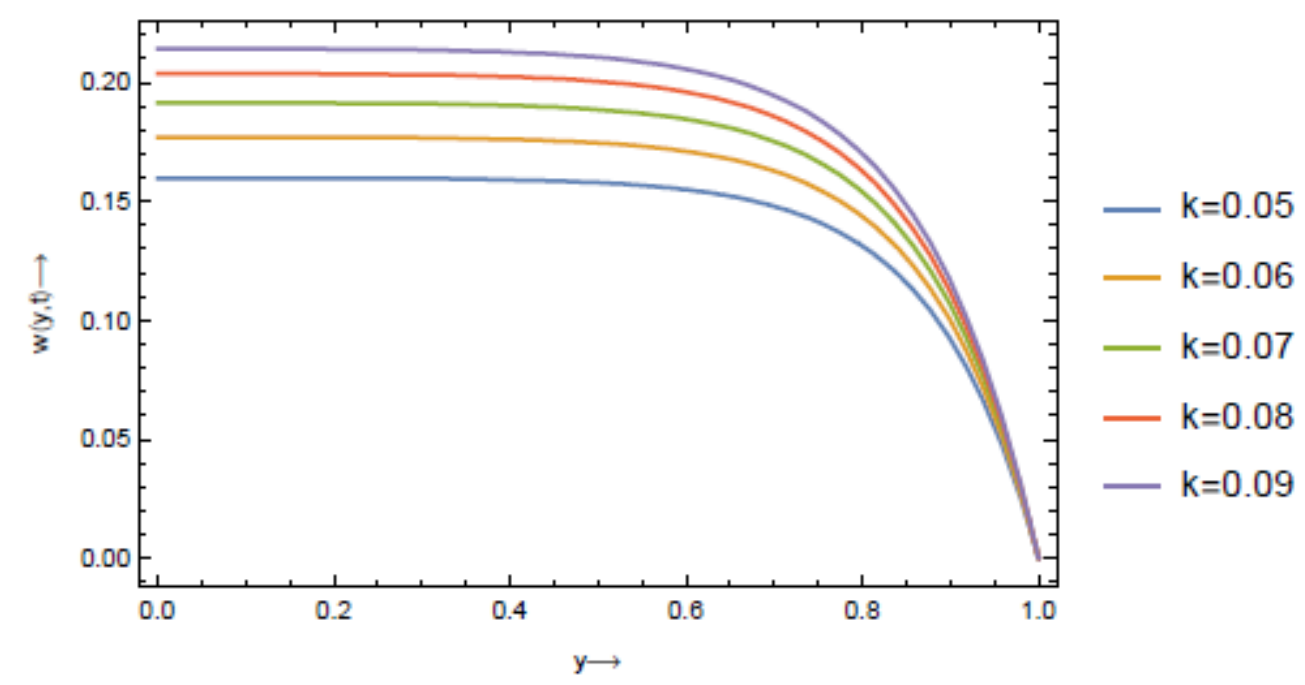

Fig. 2. Effect of permeability parameter on velocity profile $w(y, t)$, with $M=3, \alpha=0.2, \omega=3, \varepsilon=0.02, \operatorname{Pr}=21, R d_{1}=0.4, S_{2}=5, t=5 \operatorname{Re}=0.5, R_{T}=0.5, G r=15$ 


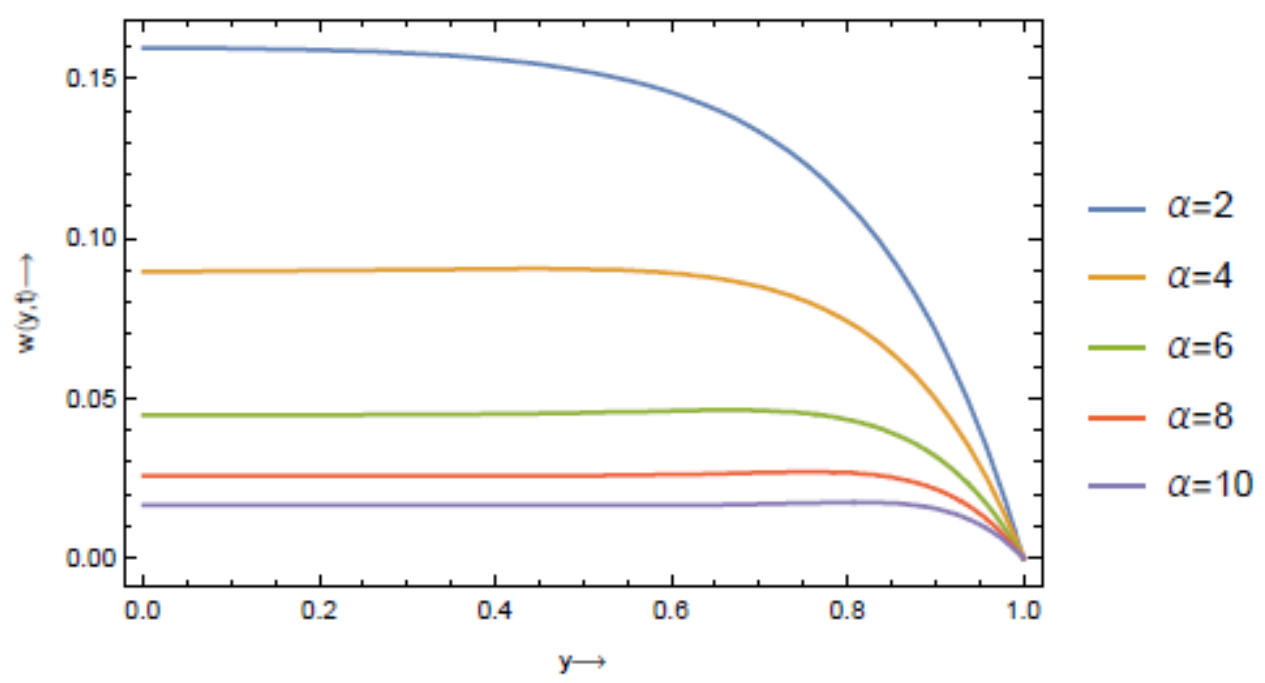

Fig. 3. Effect of womersley number on Velocity Profile $w(y, t)$, with $k=0.05, M=3, \omega=3, \varepsilon=0.02, \operatorname{Pr}=21, R d_{1}=0.4, S_{2}=5, t=5 \operatorname{Re}=0.5, R_{T}=0.5, G r=15$

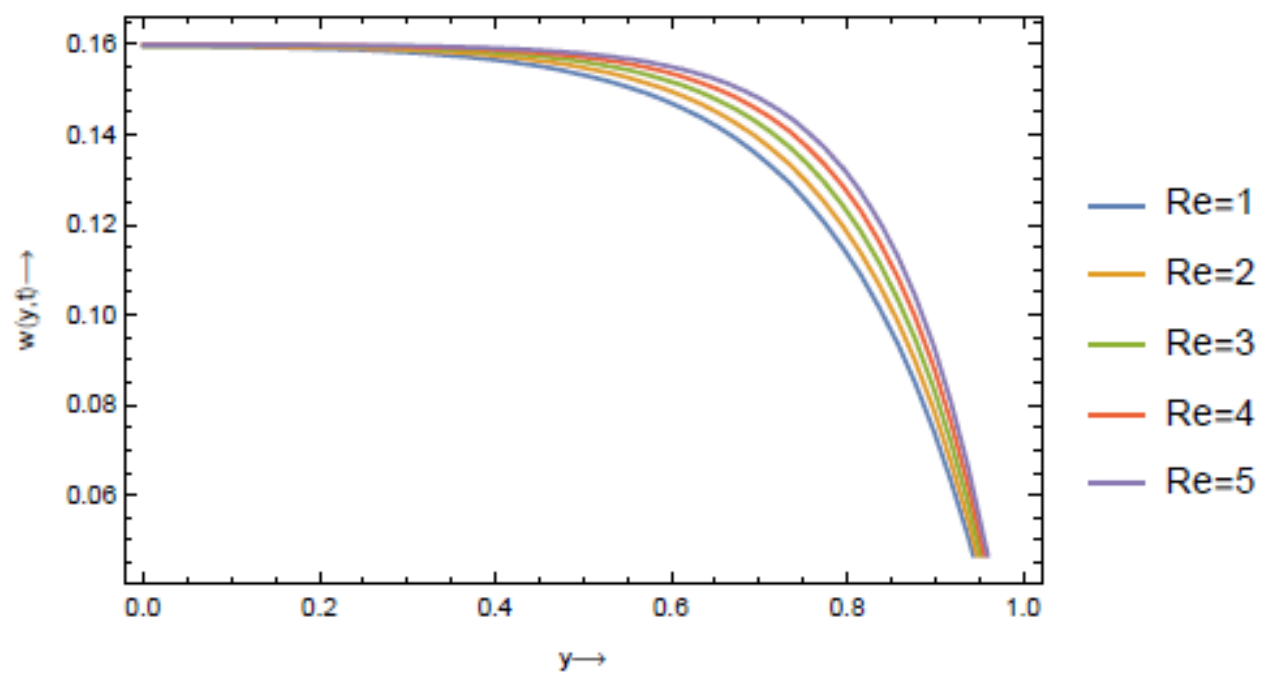

Fig. 4. Effect of Reynolds number on velocity profile $w(y, t)$, with $k=0.05, \alpha=0.2, \omega=3, \varepsilon=0.02, \operatorname{Pr}=21, R d_{1}=0.4, S_{2}=5, t=5 \quad M=3, R_{T}=0.5, G r=15$

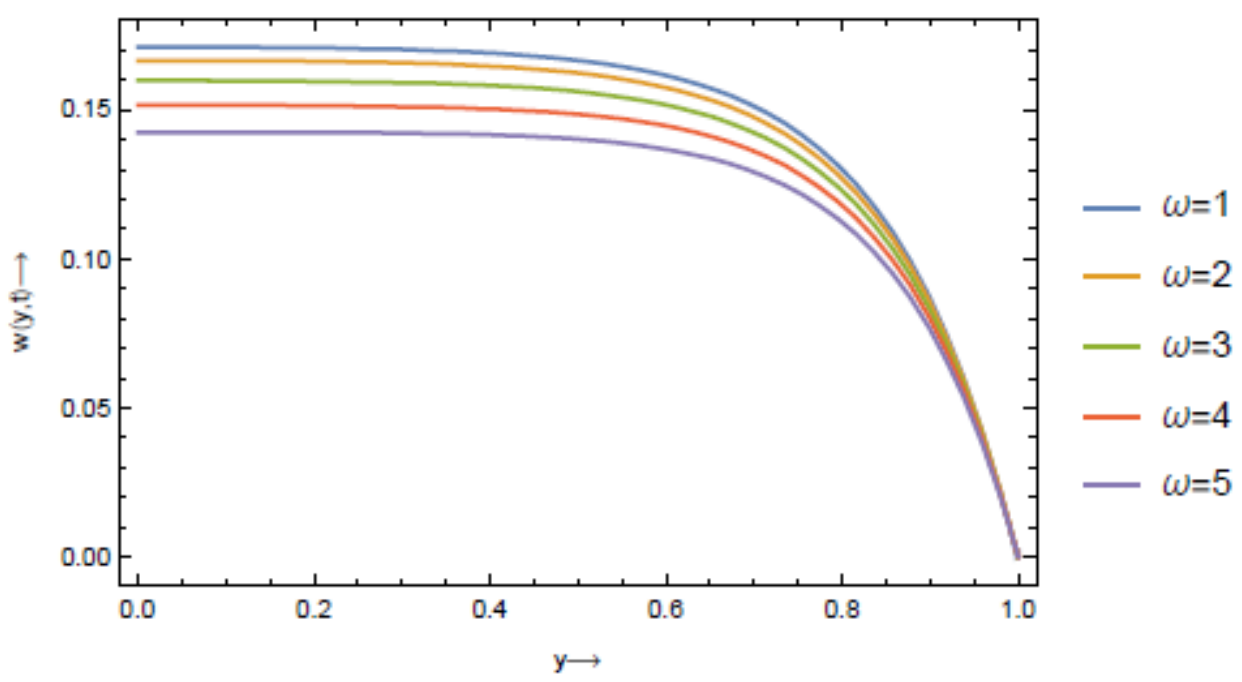

Fig. 5. Effect of oscillatory frequency on velocity profile $w(y, t)$, with $k=0.05, \alpha=0.2, M=3, \varepsilon=0.02, \operatorname{Pr}=21, R_{1}=0.4, S_{2}=5, t=5 \operatorname{Re}=0.5, R_{T}=0.5, G r=15, \omega=3$ 


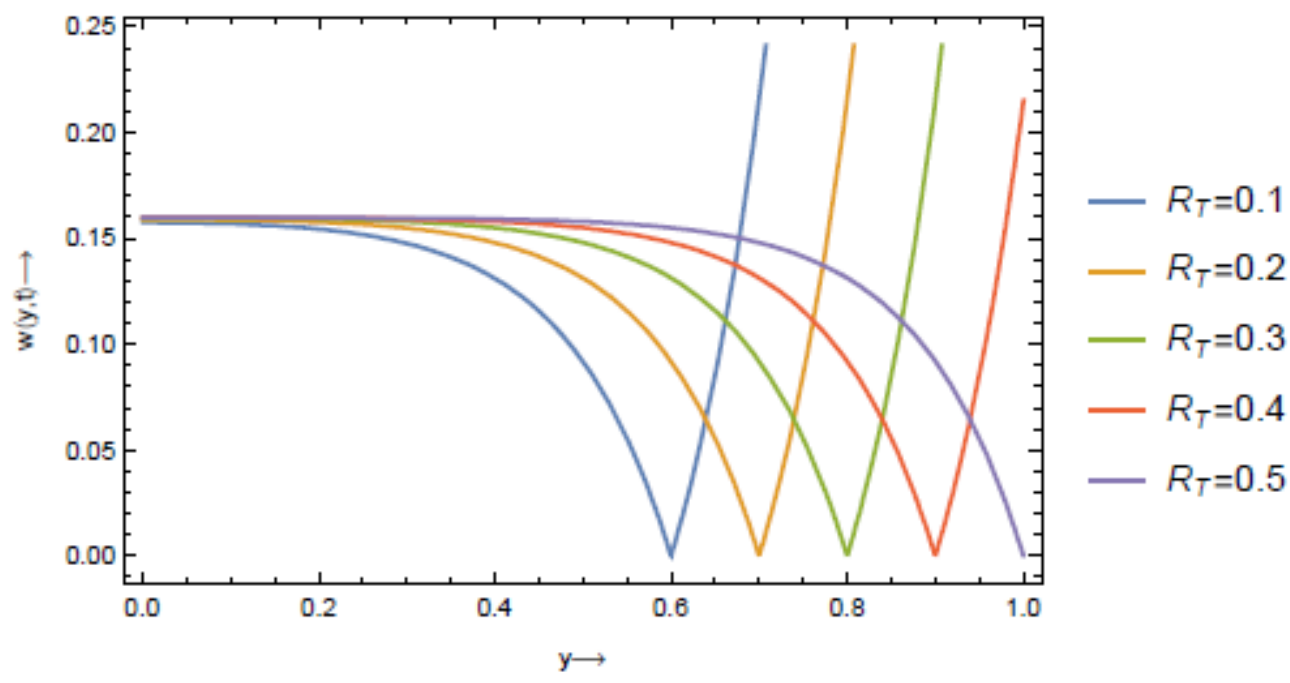

Fig. 6. Effect of treatment values on velocity profile $w(y, t)$, with $k=0.05, \alpha=0.2, M=3, \varepsilon=0.02, \operatorname{Pr}=21, R_{1}=0.4, S_{2}=5, t=5 \operatorname{Re}=0.5, G r=15, \omega=3$

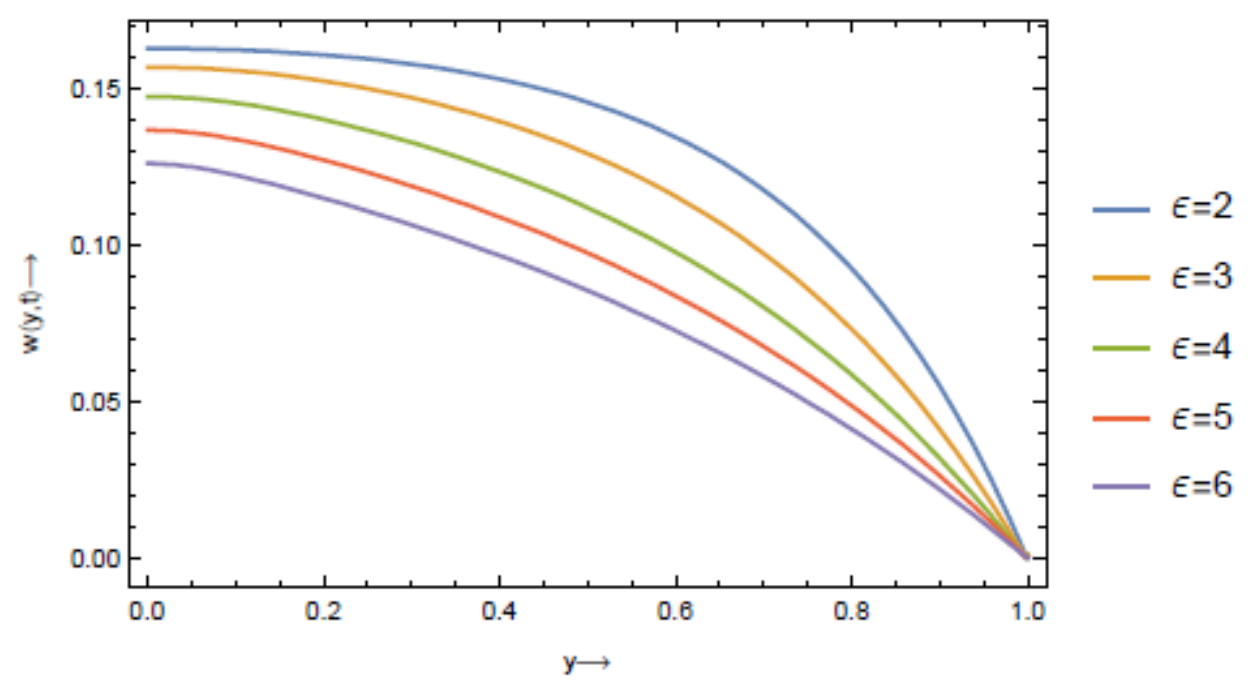

Fig. 7. Effect of perturbation parameter on velocity profile $w(y, t)$, with $k=0.05, \alpha=0.2, \operatorname{Re}=0.5, R_{T}=0.5, M=3, P r=21, G r=15 R d_{1}=0.4, S_{2}=5, t=5, \omega=3$



Fig. 8. Effect of Grashof number on velocity profile $w(y, t)$, with $k=0.05, \alpha=0.2, \operatorname{Re}=0.5, R_{T}=0.5, M=3, \operatorname{Pr}=21, R d_{1}=0.4, S_{2}=5, t=5, \varepsilon=0.02, \omega=3$ 


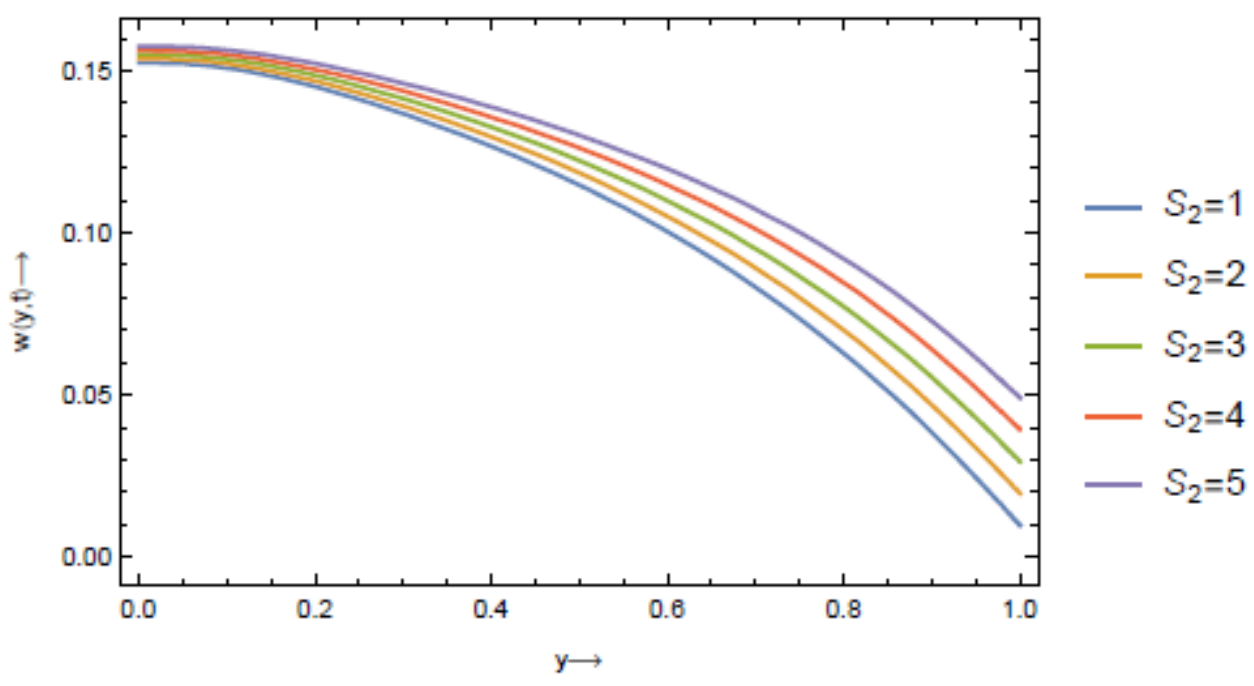

Fig. 9. Effect of Metabolic heat on velocity profile $w(y, t)$, with

$k=0.05, \alpha=0.2, \operatorname{Re}=0.5, R_{T}=0.5, M=3, \varepsilon=0.02, \operatorname{Pr}=21, R d_{1}=0.4, G r=15, t=5, \omega=3$



Fig. 10. Effect of metabolic heat on temperature profile $\theta(y, t)$, with $\operatorname{Pr}=21, \alpha=0.2, R d_{1}=0.3, R_{T}=0.5, t=5, \omega=5$

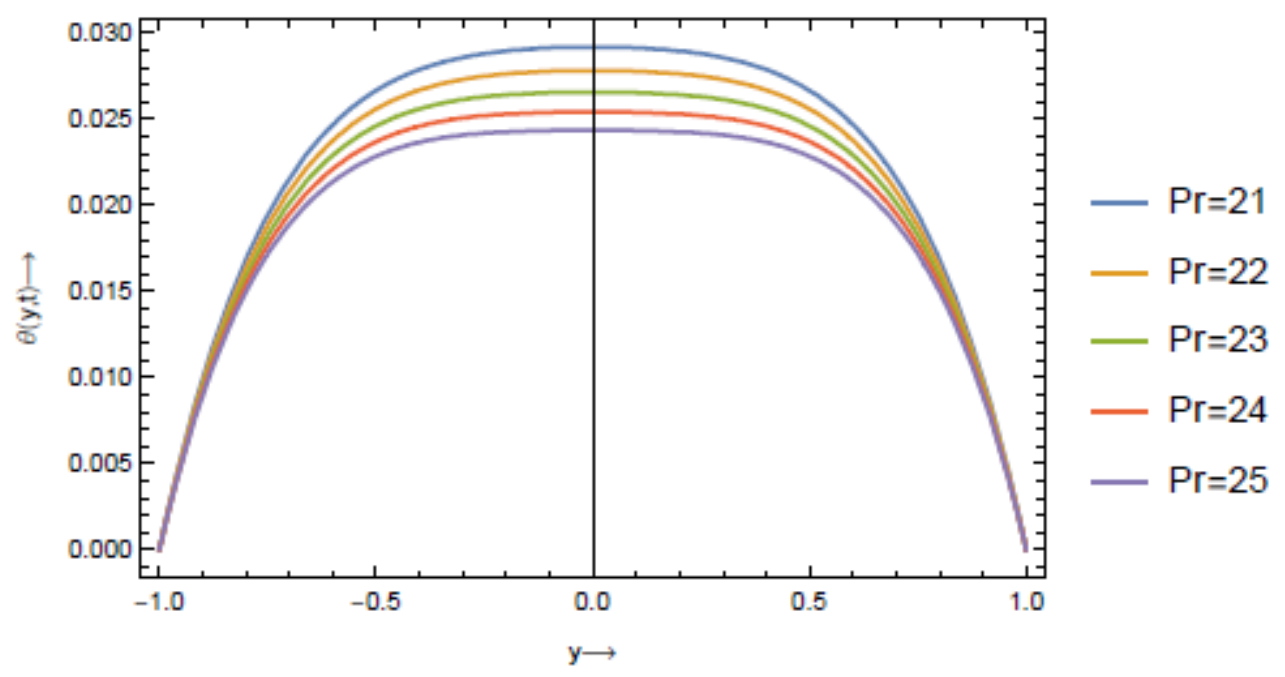

Fig. 11. Effect of Prandtl number on temperature profile $\theta(y, t)$, with $S_{2}=0.5, \alpha=0.4, R d_{1}=0.3, R_{T}=0.5, t=5, \omega=5$ 


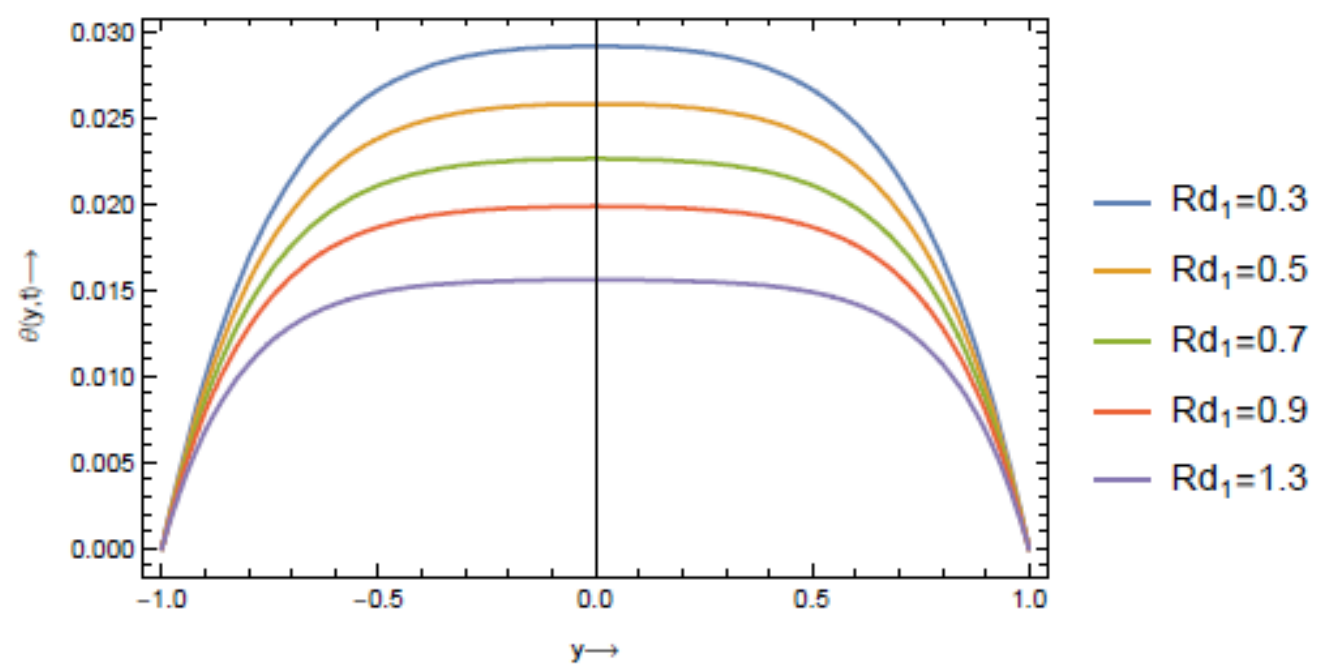

Fig. 12. Effect of heat source values on temperature profile $\theta(y, t)$, with $\operatorname{Pr}=21, \alpha=0.4, S_{2}=0.5, \omega=5, R_{T}=0.5, t=5$

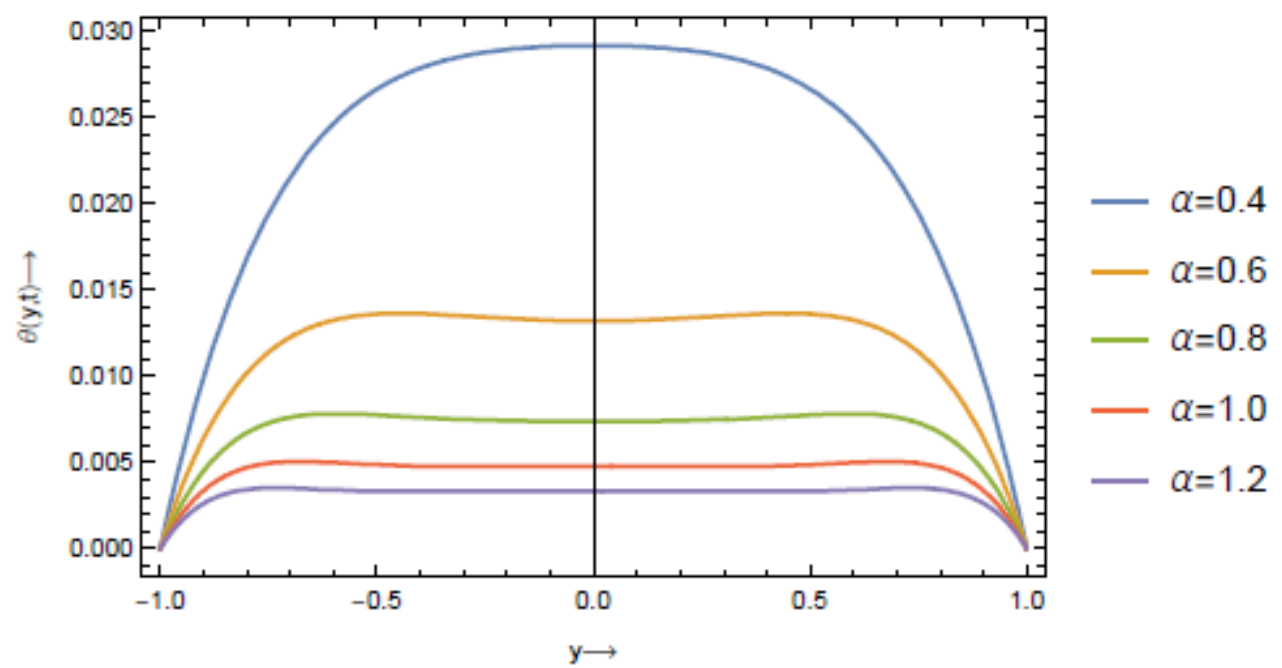

Fig. 13. Effect of womersley number on temperature profile $\theta(y, t)$, with $\operatorname{Pr}=21, \omega=5, R d_{1}=0.3, S_{2}=0.5, R_{T}=0.5, t=5$

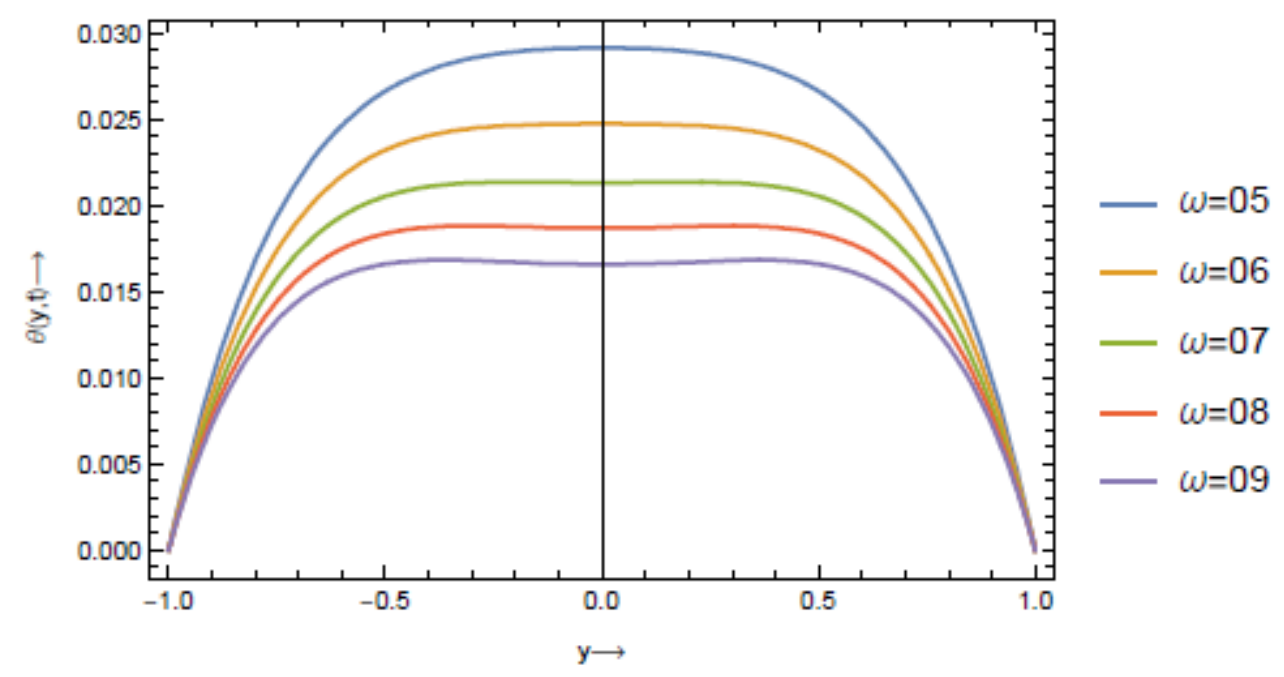

Fig. 14. Effect of oscillatory frequency on temperature profile $\theta(y, t)$, with $\operatorname{Pr}=21, \alpha=0.2, \operatorname{Rd}_{1}=0.3, S_{2}=0.5, R_{T}=0.5, t=5$

\section{DISCUSSION}

It is seen in Fig. 1 that the blood velocity profile is maximum at the centre for different values of magnetic field intensity. However, the velocity profile decreases for increasing values of magnetic field 
intensity $M=1,2,3,4,5$, while other parameters remain constant $k=0.05, \quad \alpha=0.2, \omega=3, \varepsilon=0.02$, $\operatorname{Pr}=21, \quad R d_{1}=0.4, S_{2}=5, t=5 R e=0.5, R_{T}=0.5, G r=15$. This decrease is caused by the Lorentz force due to the interaction of the magnetic field with an electrically conducting fluid. Fig. 2 depicts an increase in the blood velocity profile, for various increases in porosity parameter with values at $k=0.05,0.06,0.07,0.08,0.09$ with other parameters with values $M=3, \quad \alpha=0.2, \quad \omega=3, \quad \varepsilon=0.02$, $\operatorname{Pr}=21, R d_{1}=0.4, S_{2}=5, t=5 R e=0.5, R_{T}=0.5, G r=15$. This result is of the view that the larger the vessel matrix the better flow behavior and that is an indication of the impact of treatment, heat source. The womersley number effect was investigated and the graphical result is shown in Fig. 3. The result shows a decrease in blood velocity for the various womersley numbers $\alpha=2,4,6,8,10$, and the involvement of the other pertinent parameters $k=0.05, M=3, \omega=3, \quad \varepsilon=0.02, R e=0.5, \quad R_{T}=0.5$, $\operatorname{Pr}=21, R d_{1}=0.4, S_{2}=5, t=5$.

Fig. 4 depicts the maximum value of the velocity at the centerline of the channel, which decreases closer to the wall. It is noticed that the velocity profile increases for different values of the Reynolds number while other entering parameters values are kept as shown in Fig. 4, and the result of this investigation is consistent with those obtained by Eldesoky [27].

Fig. 5 illustrates the effect of oscillatory parameters on blood velocity. It is seen that the blood velocity profile decreases with the increase in oscillatory frequency $\omega=1,2,3,4,5, k=0.05, \alpha=0.2, \quad M=3$, $\varepsilon=0.02, \operatorname{Pr}=21, R d_{1}=0.4, S_{2}=5, t=5, \operatorname{Re}=0.5, R_{T}=0.5, G r=15, \omega=3$. This result is consistent with Those obtained by Bunonyo and Amos [2]. Fig. 6 depicts blood flow through the channel without treatment; it shows that a tumor in the human body without treatment could lead to an unorganized pattern as shown in the figure.

The sensitivity of the treatment parameter on blood velocity profile is seen in Fig. 6, and the figure depicts the behavior of the various treatment $R_{T}=0.1,0.2,0.3,0.4,0.5$ effects on the velocity profile. This result is also supported by the contribution of the other pertinent parameters values $k=0.05, \alpha=0.2, M=3, \varepsilon=0.02, \operatorname{Pr}=21, R d_{1}=0.4, \operatorname{Re}=0.5, G r=15, \omega=3, S_{2}=5, t=5$

It is seen in Fig. 7 to 9, which depict the effect of perturbed parameter value, Grashof number and metabolic heat on velocity profile respectively. The figures showed that blood velocity profile increases for the increasing values $\varepsilon=2,3,54,5,6, G r=5,10,15,20,25$ and $S_{2}=1,2,3,4,5$, and $k=0.05$, $\alpha=0.2, \quad \operatorname{Re}=0.5, R_{T}=0.5, M=3, R d_{1}=0.4, G r=15, t=5, \omega=3, \varepsilon=0.02, \operatorname{Pr}=21$. Fig. 10 illustrates that blood temperature increases for the increasing values of the metabolic heat on temperature profile $S_{2}=0.5,0.7,0.9,1.2,1.5$, with some of the pertinent parameters values $\operatorname{Pr}=21, \alpha=0.2$, $R d_{1}=0.3, R_{T}=0.5, t=5, \omega=5$. In a similar vein, in Fig. 12, the temperature increasing for an increasing values of heat source $R d_{1}=0.3,0.5,0.7,0.9,1.2$, with some of the pertinent parameters values $\operatorname{Pr}=21, \alpha=0.4, S_{2}=0.5, \omega=5, R_{T}=0.5, t=5$. However, it is seen in Fig. 11, and Figs. 13-14, that the temperature profiles decrease with the increasing values of Prandtl number, Womersley number, and oscillatory frequency parameters, respectively, while parameter values, $S_{2}=0.5, \alpha=0.4, R d_{1}=0.3, R_{T}=0.5, t=5, \omega=5$ also contributes to the aforementioned results.

\section{CONCLUSION}

After careful formulation of the mathematical models, analytically solved solutions, and numerical simulation using Wolfram Mathematica software, we conclude as follows:

1. The blood velocity decreases with an increase in magnetic field intensity, where the velocity reaches different peaks, 2.10491, 1.60806, 1.15312, 0.82538 and 0.603835 respectively, before it began to decrease to zero when boundary thickness is large. The results are in good agreement with studies carried out by Shit and Roy [29].

2. It is observed that blood velocity increases with increasing values of the permeability parameter $k=0.05,0.06,0.07,0.08,0.09$.

3. It is noticed that blood velocity decreases for the various values of the Womersley number $\alpha=2,4,6,8,10$ while other pertinent parameter values are kept constant.

4. The Reynolds number increase caused the blood velocity to increase and attain a common peak before dropping to zero as the boundary layer gets thicker. The result of the investigation is consistent with that obtained by Eldesoky [27]. 
5. The treatment parameter increase causes an increase in blood velocity, but we noticed a decrease in velocity for the increase in the oscillatory frequency parameter $\omega=1,2,3,4,5$.

6. The research also investigated the impact of the perturbation parameter variation on the velocity profile. It was noticed that the velocity profile decreases with an increase in the perturbation parameter value $\varepsilon=2,3,4,5,6$.

7. The buoyancy parameter was investigated to note the fact that the velocity profile increases for an increase in the Grashof number $G r=5,10,15,20,25$. It is also observed that as the metabolic heat parameter increases, so does the blood velocity profile $S_{2}=1,2,3,4,5$. The blood temperature profile was also noticed to increase for an increase in metabolic heat parameters with values $S_{2}=0.5,0.7,0.9,1.2,1.5$.

8. The effect of the Prandtl number values $\operatorname{Pr}=21,22,23,24,25$ The velocity of blood was investigated, and it was noticed that the velocity decreased with an increase in Prandtl number.

9. The temperature profile was investigated for an increase in heat source parameter values $R d_{1}=0.3,0.5,0.7,0.9,1.3$, It is seen that the temperature profile increases with an increase in heat source, and this increase helps in facilitating blood flow through the microchannel.

10. The womersley number $\alpha=0.4,0.6,0.8,1.0,1.2$ In a similar vein, the variation in oscillatory frequency parameter is seen to have caused a decrease in blood temperature $\omega=05,06,07,08,09$ This resulted in a decrease in blood temperature profile. This result is consistent with those obtained by Bunonyo and Amos [2]..

\section{REFERENCES}

[1] Ghanem F, Vodnala D, Kalavakunta JK, Durga S, Thormeier N, Subramaniyam P, Abela S, Abela GS. Cholesterol crystal embolization following plaque rupture: a systemic disease with unusual features. Journal of biomedical research, 2017;31:8294.

[2] Bunonyo KW, Amos E. Lipid Concentration Effect on Blood Flow Through an Inclined Arterial Channel with Magnetic Field. Mathematical Modelling and Applications. 2020;5:129-137.

[3] Davies O. Okpeta \& K.W. Bunonyo. Effect of Lipoprotein Concentration on MHD Blood flow through Parallel Plate with Heat source and Magnetic Intensity International Journal of Mathematics Trends and Technology, 2021;67:62-80.

[4] Singh J, Rathee R. Analytical solution of two-dimensional model of blood flow with variable viscosity through an indented artery due to LDL effect in the presence of magnetic field. International Journal of Physical Sciences, 2010;5:1857-68.

[5] Cabrales LE, Aguilera AR, Jiménez RP, Jarque MV, Ciria HM, Reyes JB, Mateus MA, Palencia FS, Ávila MG. Mathematical modeling of tumor growth in mice following low-level direct electric current. Mathematics and Computers in Simulation, 2008;78:112-20.

[6] Kumar S, Atangana A. A numerical study of the nonlinear fractional mathematical model of tumor cells in presence of chemotherapeutic treatment. International Journal of Biomathematics, 2020;13:2-15.

[7] Gholami A, Mang A, Biros G. An inverse problem formulation for parameter estimation of a reaction-diffusion model of low grade gliomas. Journal of mathematical biology, 2016;72:409-33.

[8] Molloy J, Markus HS. Asymptomatic embolization predicts stroke and TIA risk in patients with carotid artery stenosis. Stroke. 1999;30:1440-3

[9] Bru A, Herrero MA. From the physical laws of tumor growth to modelling cancer processes. Mathematical Models and Methods in Applied Sciences, 2006;16:1199-218.

[10] Dai F, Liu B. Optimal control and pattern formation for a haptotaxis model of solid tumor invasion. Journal of the Franklin Institute, 2019;356:9364-406.

[11] Düchting W, Vogelsaenger T. Aspects of modelling and simulating tumor growth and treatment. Journal of Cancer Research and Clinical Oncology, 1983;105:1-2.

[12] Düchting W, Vogelsaenger T. Recent progress in modelling and simulation of three-dimensional tumor growth and treatment. Biosystems, 1985;18:79-91.

[13] Düchting W, Vogelsaenger T. Simulation of three-dimensional cell growth with application to optimal tumor treatment. Annual Review in Automatic Programming, 1985;12:439-42.

[14] Hawkins-Daarud A, Rockne RC, Anderson AR, Swanson KR. Modeling tumor-associated edema in gliomas during antiangiogenic therapy and its impact on imageable tumor. Frontiers in oncology, 2013;3:1-8.

[15] Makinde T, Murphy RF, Agrawal DK. Immunomodulatory role of vascular endothelial growth factor and angiopoietin-1 in airway remodeling. Current molecular medicine, 2006;6:831-41.

[16] Makinde OD, Osalusi E. MHD steady flow in a channel with slip at the permeable boundaries. Romanian Journal of Physics, 2006;51:319-328.

[17] Mandal PK. An unsteady analysis of non-Newtonian blood flow through tapered arteries with a stenosis. International journal of non-linear mechanics, 2005;40:151-64.

[18] Unni, P., \& Seshaiyer, P. Mathematical modeling, analysis, and simulation of tumor dynamics with drug interventions. Computational and mathematical methods in medicine, 2019;2019:1-13.

[19] Miklavčič D, Jarm T, Karba R, Serša G. Mathematical modelling of tumor growth in mice following electrotherapy and bleomycin treatment. Mathematics and computers in simulation, 1995;39:597-602.

[20] Ansarizadeh F, Singh M, Richards D. Modelling of tumor cells regression in response to chemotherapeutic treatment. Applied Mathematical Modelling. 2017;48:96-112.

[21] Morales-Delgado VF, Gómez-Aguilar JF, Saad K, Escobar Jiménez RF. Application of the Caputo-Fabrizio and Atangana-Baleanu fractional derivatives to mathematical model of cancer chemotherapy effect. Mathematical Methods in the Applied Sciences. 2019;42:1167-93.

[22] Padi TR, Jayabharathiraj J, Kumar BN, Usha CL, Reddy PR. Stochastic Modelling of Tumor Growth within Organ during Chemotherapy Using Bivariate Birth, Death and Migration Processes. IOSR Journal of Mathematics, 2014;10:01-8. 
[23] Kazmi N, Hossain MA, Phillips RM, Al-Mamun MA, Bass R. Avascular tumour growth dynamics and the constraints of protein binding for drug transportation. Journal of theoretical biology, 2012;313:142-52.

[24] Zhu, Q., Zhang, A., Liu, P., \& Xu, L. X. Study of tumor growth under hyperthermia condition. Computational and mathematical methods in medicine, 2012;2012:1-9.

[25] Bunonyo KW, Amadi CU. Mathematical Modeling of Treatment Effect on Tumor Growth and Blood Flow through a Channel with Magnetic Field. JAMCS [Internet], 2021;36:66-79. https://www.journaljamcs.com/index.php/JAMCS/article/view/30385

[26] Misra JC, Adhikary SD. MHD oscillatory channel flow, heat and mass transfer in a physiological fluid in presence of chemical reaction. Alexandria Engineering Journal. 2016;55:287-97.

[27] Eldesoky IM. Mathematical analysis of unsteady MHD blood flow through parallel plate channel with heat source. International Conference on Mathematics and Engineering Physics, 2012;6:1-16.

[28] Domingues JS. Gompertz model: Resolution and analysis for tumors. Journal of Mathematical Modelling and Application, 2012;1:70-7.

[29] Shit GC, Roy M. Hydromagnetic pulsating flow of blood in a constricted porous channel: A theoretical study. In Proceedings of the World Congress on Engineering, London, 2012;1:1-6.

\section{NOMENCLATURES}

\begin{tabular}{|c|c|}
\hline$\left(u^{*}, v^{*}, w^{*}\right)$ & Dimensional Velocity components \\
\hline$y^{*}$ & Dimensional radius of the artery \\
\hline$x^{*}$ & Dimensional axial distance \\
\hline$t^{*}$ & Dimensional time \\
\hline$B_{0}$ & Magnetic field parameter \\
\hline$R(x)$ & Tumor depleted region of the channel \\
\hline$R_{0}$ & Normal region of the channel \\
\hline$R_{T}$ & Treatment parameter \\
\hline$P_{0}$ & Diastolic blood pressure \\
\hline$P_{1}$ & Systolic blood pressure \\
\hline$T_{a}$ & Arterial temperature \\
\hline$T$ & Variable temperature \\
\hline$F$ & Blood mass flow rate \\
\hline$w_{1}(y)$ & Perturbed unsteady state velocity \\
\hline$w_{0}(y)$ & Perturbed steady state velocity \\
\hline$w(y, t)$ & Fluid velocity profile \\
\hline$\vec{B}$ & Magnetic flux density \\
\hline$V_{0}$ & Suction velocity \\
\hline$S_{2}$ & Metabolic heat parameter \\
\hline$R d_{1}$ & Heat source parameter \\
\hline$G r$ & Grashof Number \\
\hline$\vec{E}$ & Electrical field intensity \\
\hline
\end{tabular}

GREEK SYMBOLS

$v$

Kinematic Viscosity of blood

$\mu_{b} \quad$ Dynamic viscosity of blood

$c_{b} \quad$ Initial height of tumor growth

$k_{T} \quad$ Initial height of tumor growth 


$\begin{array}{ll}\rho, \rho_{b} & \text { Tissue and blood densities } \\ g & \text { Acceleration due to gravity } \\ \delta_{0} & \text { Initial Area of plaque } \\ \delta & \text { Height of palque } \\ \sigma & \text { Electrical conductivity } \\ \omega & \text { Oscillatory frequency } \\ \beta_{0} & \text { Constant term due to steady state } \\ \beta_{1} & \text { Constant term due to unsteady state }\end{array}$

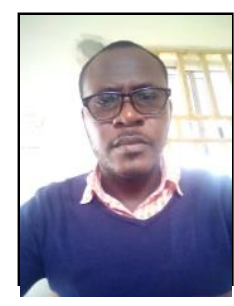

Dr. Bunonyo Wilcox Kubugha, is a reviewer/researcher/lecturer, studied Applied Mathematics at the Rivers State University, Port Harcourt, whose research interest/focus is in the areas of Haemodynamics, MHD and Mathematical Modeling, Applied Mathematics and Biostatistics. 